\title{
Wider-frequency combs generation, noise reduction, and repetition rate tuning in quantum-dot mode-locked lasers
}

\author{
T. Habruseva ${ }^{* a, b}$, D. Arsenijevic ${ }^{c}$, D. Bimberg ${ }^{c}$, G. Huyet ${ }^{a}$, S. P. Hegarty ${ }^{a}$ \\ ${ }^{a}$ CAPPA, Cork Institute of Technology and Tyndall National Institute, Cork, Ireland; \\ ${ }^{\mathrm{b}}$ Aston University, Aston Triangle, B4 7ET, Birmingham, UK; \\ 'Institut für Festkörperphysik, Technische Universität Berlin, Berlin, Germany
}

\begin{abstract}
We describe the technique allowing for generation of low-noise wider frequency combs and pulses of shorter duration in quantum-dot mode-locked lasers. We compare experimentally noise stabilization techniques in semiconductor modelocked lasers. We discuss the benefits of electrical modulation of the laser absorber voltage (hybrid mode-locking), combination of hybrid mode-locking with optical injection seeding from the narrow linewidth continues wave master source and optical injection seeding of two coherent sidebands separated by the laser repetition rate.
\end{abstract}

Keywords: Lasers and laser optics; mode-locked lasers; quantum-dot devices

\section{INTRODUCTION}

Quantum-dot mode-locked lasers (QD-MLLs) are attractive sources of sub-picosecond pulses at high repetition rates and generate coherent frequency combs in optical domain [1,2]. Optical frequency combs emitted by MLLs can be used in arbitrary waveform generation and metrology, while short periodic pulses with low jitter are required in fiber optic communication systems, optical sampling and all-optical clock recovery [3]. Passive mode-locking is more cost-efficient and robust to implement when compared with active or hybrid mode-locking. However, frequency combs generated by passively mode-locked lasers (PMLLs) often exhibit large phase noise and jitter, which makes them ineffective in highspeed applications [4]. Therefore, noise stabilization techniques are required to improve performance of QD-PMLLs.

In this paper we compare experimentally noise stabilization techniques allowing control of phase noise and frequency tuning in monolithic QD-MLLs. In particular, we discuss the benefits of electrical modulation of the laser absorber voltage (hybrid mode-locking) [5-7], combination of hybrid mode-locking with optical injection seeding from the narrow linewidth continues wave (CW) master source and optical injection seeding of two coherent sidebands separated by the laser repetition rate [8]. For this study all measurements were performed on the same device, a $40 \mathrm{GHz} \mathrm{InAs} / \mathrm{GaAs} \mathrm{QD}$ MLL, under the same temperature and bias conditions. We use the benefits of coherent sidebands injection to develop novel technique for synchronization of QD-MLLs outputs, which allows generation of wider coherent frequency combs with reduced phase noise.

\section{MEASUREMENTS}

Monolithic two-section QD lasers emitting at $1.3 \mu \mathrm{m}$ were used in these studies. The active zone of the devices consisted of InAs QDs embedded in InGaAs quantum wells. The comparison of noise stabilization techniques was performed on the QD device with $1 \mathrm{~mm}$ length (corresponding pulse repetition rate, $F_{\text {rep }}$, was $39.5 \mathrm{GHz}$ ) and with $10 \%$ absorber section. The facet next to the absorber was coated for high reflectivity of $95 \%$; the facet next to the gain section was as cleaved $(\mathrm{R} \sim 30 \%)$. All experimental investigations were performed under the same temperature of $21^{\circ} \mathrm{C}$ and similar bias conditions: the absorber section reverse bias was $-8.0 \mathrm{~V}$ and the gain current was around 50-60 $\mathrm{mA}$. The laser output was characterized via optical and electrical spectrum analyzers and an autocorrelator.

For the hybrid mode-locking, the absorber bias was modulated at the frequency close to $39.5 \mathrm{GHz}$ via a low noise Agilent signal generator, similar to Ref. 7. The frequency and the amplitude of the modulation were varied. We used CW tunable laser source with a narrow optical linewidth of $\sim 100 \mathrm{kHz}$ for the optical injection. For coherent sidebands injection, the light from the tunable laser was modulated by a Mach-Zehnder amplitude modulator. The modulator was biased at the transmission minimum and driven via Agilent signal generator at half repetition rate, giving two coherent

Semiconductor Lasers and Laser Dynamics VI, edited by Krassimir Panajotov, Marc Sciamanna,

Angel Valle, Rainer Michalzik, Proc. of SPIE Vol. 9134, 91340F · @ 2014 SPIE

CCC code: $0277-786 X / 14 / \$ 18 \cdot$ doi: $10.1117 / 12.2052351$

Proc. of SPIE Vol. $913491340 \mathrm{~F}-1$ 
sidebands separated by $F_{\text {rep }}$ with a suppressed carrier frequency. The polarization of the injected light was aligned with the TE mode of the QD laser.

In the passive mode-locked regime, the QD-MLL emitted pulses of 2.8 ps duration. The pulse train demonstrated large timing jitter values of 9 ps and significant phase noise with optical modal linewidth values in the range of $100 \mathrm{MHz}$ [9]. The jitter was retrieved from single-sideband noise measured via electrical spectrum analyzers and integrated from 10 $\mathrm{kHz}$ to $1 \mathrm{GHz}$.

\section{NOISE REDUCTION AND REPETITION RATE TUNING}

The noise stabilization techniques have been compared: hybrid mode-locking, All studied stabilization techniques resulted in the significant timing jitter reduction compared to passively MLL and allowed tuning of the device repletion rate. With hybrid mode-locking we achieved a 14-times jitter reduction and laser repetition rate tuning over the $10 \mathrm{MHz}$ range, however, it did not affect device phase noise and pulse chirp [6-7]. Both hybrid mode-locking with optical injection and coherent sidebands injection allowed laser repetition rate tuning, jitter reduction, and also resulted in the phase noise reduction and red-shifted narrowed optical spectrum.

The outcomes of combination of hybrid mode-locking with CW optical injection seeding are summarized in Table 1. With injection seed the integrated jitter was reduced to $240 \mathrm{fs}$ and the repetition rate tuning range was increased to 167 MHz [9]. Both, optical injection power and modulation power influenced the results.

Table 1. Characteristics of the QD-MLL with hybrid mode-locking and optical injection.

\begin{tabular}{|l|l|l|c|c|}
\hline$P_{\text {inj; }}, \mu W$ & $\lambda_{\text {inj, }}, \mathrm{mm}$ & $P_{\text {mod }}, \mathrm{dBm}$ & $\begin{array}{c}\text { Tuning range, } \\
\text { MHz }\end{array}$ & $\begin{array}{c}\text { Integrated } \\
\text { jitter, } f s\end{array}$ \\
\hline 770 & 1288.44 & 14 & 167 & 240 \\
770 & 1288.44 & 9 & 40 & 365 \\
440 & 1285.33 & 5 & 18 & 793 \\
440 & 1285.33 & 3 & 8.9 & 885 \\
440 & 1285.33 & 0 & 8.1 & 1658 \\
\hline
\end{tabular}

Remarkably, injection locking could result in the shift of the pulse repetition rate to the both higher and smaller frequencies, subject to the parameters, such as injected power, gain current and modulation amplitude. This opens possibilities for the cleaving errors compensation in either side as well as widens the summarized locking range to 327 $\mathrm{MHz}$, as shown in Figure 1.

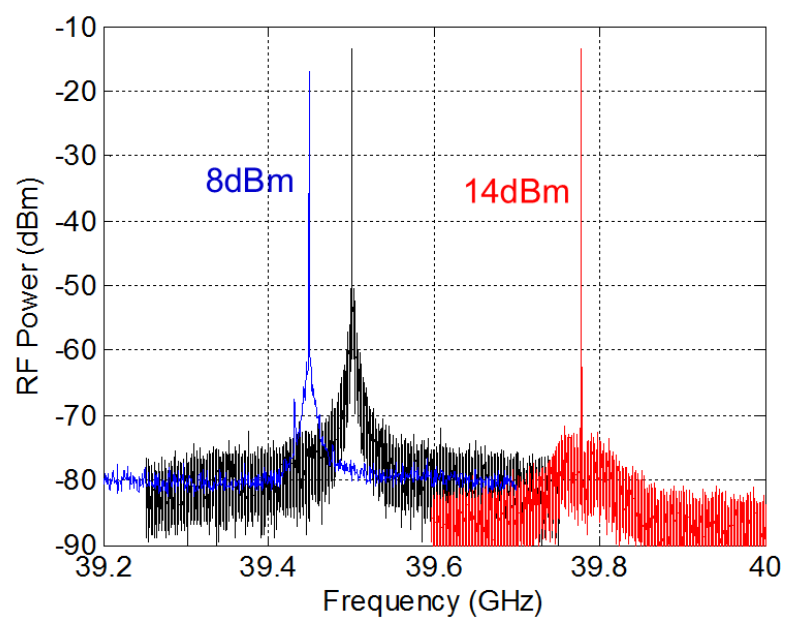

Figure 1. RF spectra of the QD-MLL in passive mode-locked regime (black), with hybrid mode-locking and optical injection seeding for $8 \mathrm{dBm}$ (blue) and $14 \mathrm{dBm}$ (red) modulation power. Gain current: $60 \mathrm{ma}$, absorber bias: $-8.0 \mathrm{~V}$. 
We achieved the lowest integrated jitter of $121 \mathrm{fs}$ and the widest frequency tuning range of $342 \mathrm{MHz}$ with coherent sidebands injection seeding of the QD-PMLLs [9]. The sidebands injection also resulted in all-modal linewidth reduction, optical spectrum narrowing and its red shift from the injection seed [10-12]. The outcomes of the sidebands injection seeding are summarized in Table 2. The lowest integrated jitter and the widest frequency tuning range were achieved for higher injection power, due to optical spectrum narrowing and fewer modes in the optical spectrum. However, spectral width reduction leaded to the pulse width broadening from 3 to $5.2 \mathrm{ps}$.

Table 2. Characteristics of the QD-MLL with sidebands injection locking.

\begin{tabular}{|l|l|c|c|}
\hline$P_{i n j}, \mu W$ & $\lambda_{i n j}, n m$ & $\begin{array}{c}\text { Tuning range, } \\
M H z\end{array}$ & $\begin{array}{c}\text { Integrated } \\
\text { jitter, } f S\end{array}$ \\
\hline 570 & 1278.9 & 342 & 121 \\
433 & 1278.9 & 198 & 177 \\
385 & 1278.9 & 3.0 & 1596 \\
304 & 1278.9 & 1.8 & 1656 \\
240 & 1278.9 & 1.2 & 1850 \\
\hline
\end{tabular}

\section{COMBINATION OF QD-MLL OUTPUTS}

When QD-MLL was locked to the tunable master source, its optical spectrum was narrowed with a major power red shifted from the injection wavelength [11, 12]. We used this optical spectrum red shift to generate highly coherent wider frequency combs. We used two $10 \mathrm{GHz}$ QD-MLLs made from the same wafer and cleaved together for the synchronization [11]. When the first QD-MLL was injection-locked to the coherent sidebands, it resulted in the laser optical spectrum narrowing and red-shift from the injection as shown in Figure 2 (a, blue). The injection wavelength is shown with a black arrow. The sideband injection locking also resulted in reduction of jitter and optical linewidth, as previously discussed [12]. Then, two modes apart from the injection wavelength were filtered from the first laser and coupled into the second QD-MLL. The filtered modes are shown in black in $(a, b)$. When the second laser was locked to the filtered modes, its noise was also reduced and optical spectrum (b, red) shifted to the longer wavelength from the injected modes (b, black). The outputs from the both lasers were recombined resulting in a wider optical spectrum due to the consequent spectral red-shifts of the devices Figure 2 (c).

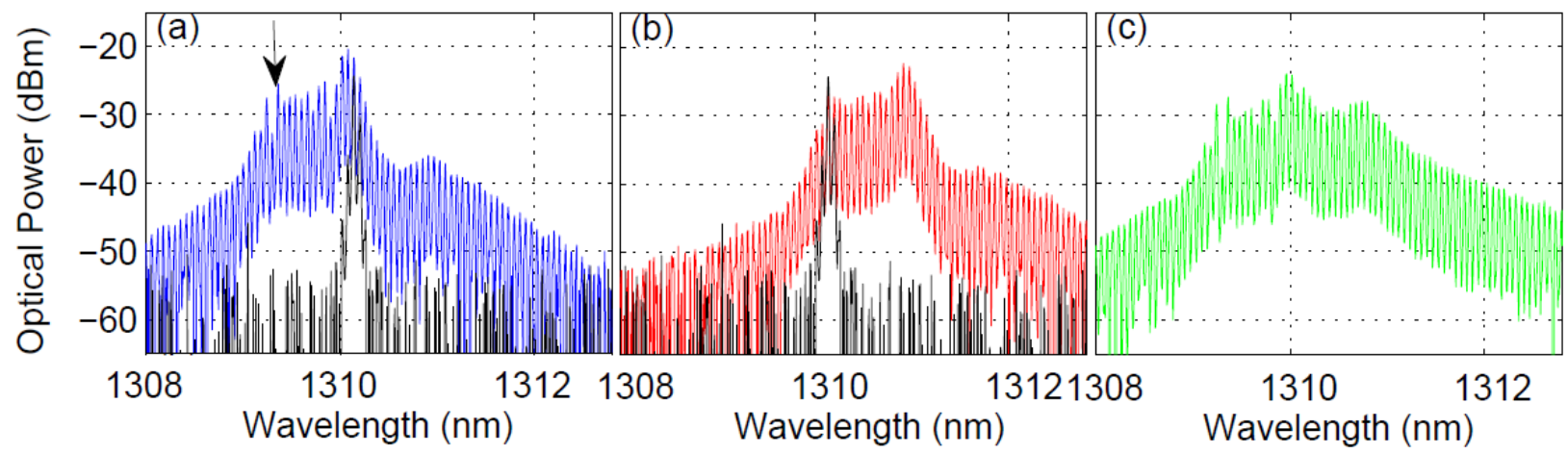

Figure 2. (a) Optical spectra of sidebands injection-locked laser 1 (blue), filtered modes (black), and injection seed (an arrow). (b) Optical spectra of the filtered modes (black) and laser 2 (red), locked to the modes. (c) Combined optical spectra of the lasers 1 and 2 (green). Laser 1 gain current: $216 \mathrm{~mA}$; absorber bias: $-2 \mathrm{~V}$. Laser 2 gain current: $211 \mathrm{~mA}$, absorber bias: $-2 \mathrm{~V}$. 


\section{CONCLUSION}

We compared experimentally noise stabilization techniques in the monolithic QD-MLL. We discussed the benefits of hybrid mode-locking, combination of hybrid mode-locking with optical injection seeding and optical injection seeding of two coherent sidebands separated by the laser repetition rate. For this study, all measurements were performed on the same device, a $40 \mathrm{GHz}$ InAs/GaAs QD-MLL, under the same temperature and bias conditions.

We used the benefits of coherent sidebands injection-locking to develop technique for producing wider coherent frequency combs. We demonstrated the generation of highly coherent wider frequency comb from two synchronized 10 GHz QD-MLLs. The combined frequency comb has the benefits of the sideband injected laser in terms of jitter and phase noise reduction.

\section{ACKNOWLEDGMENTS}

This work was funded by the Marie Curie Action FP7-PEOPLE-2011-IEF, HARMOFIRE project, Grant No 299288, by the EU FP7 Marie Curie Action FP7-PEOPLE-2010-ITN, PROPHET project, Grant No. 264687, by the INSPIRE programme, funded by the Irish Government's Programme for Research in Third Level Institutions, Cycle 4, National Development Plan 2007-2013, by Science Foundation Ireland under Contract No. 07/IN.1/I929 and from the DFG in the framework of the SFB 787.

*tatiana.gabruseva@gmail.com

\section{REFERENCES}

[1] Rafailov, E. U., Cataluna, M. A. and Sibbett, W., "Mode-locked quantum dot lasers," Nat. Photonics 1, 395-401 (2007).

[2] Thompson, M. G., Rae, A. R., Xia, M., Penty, R. V., and White, I. H., "InGaAs quantum-dot mode-locked laser diodes," IEEE J. Sel. Topics Quantum Electron. 15 (3), 661- 672 (2009).

[3] Silva, M. S., Lagrost, A., Bramerie, L., Gay, M., Besnard, P., Joindot, M., Simon, J.-C., Shen, A., and Duan, G.H., "Up to $427 \mathrm{GHz}$ all optical frequency down-conversion clock recovery based on quantum-dash Fabry-Perot mode-locked laser," J. Lightwave Technol., 29 (4), 609 - 615 (2011).

[4] Lin, Ch.-Y., Grillot, F., Li, Y., Raghunathan, R., and Lester, L. F., "Characterization of timing jitter in a 5 Ghz quantum dot passively mode-locked laser," Opt. Express, 18(21), 21932-21937 (2010).

[5] Thompson, M. G., Marinelli, C., Tan, K. T., Williams, K. A., Penty, R. V., White, I. H., Kaiander, I. N., Sellin, R. L., Bimberg, D., Kang, D. J., Blamire, M. G., Visinka, F., Jochum, S., and Hansmann S., "10 GHz hybrid modelocking of monolithic InGaAs quantum dot lasers," Electron. Lett. 39, 1121 (2003).

[6] Thompson, M. G., Larsson, D., Rae, A., Yvind, K., Penty, R. V., White, I. H., Hvam, J., Kovsh, A. R., Mikhrin, S. S., and Krestnikov, I. L., "Monolithic hybrid and passive mode-locked $40 \mathrm{GHz}$ quantum dot laser diodes ," Proc. ECOC (2006).

[7] Fiol, G., Arsenijevic, D., Bimberg, D., Vladimirov, A. G., Wolfrum, M., Viktorov, E. A., and Mandel, P., Appl. Phys. Lett. 96, 011104 (2010).

[8] Goldberg, L., Taylor H. F., and Weller, J. F., "FM sideband injection locking of diode lasers," Electron. Lett. 18 (11), 1019 - 1020 (1982).

[9] Habruseva, T., Arsenijević, D., Kleinert, M., Bimberg, D., Huyet, G., and Hegarty, S. P., "Optimum phase noise reduction and repetition rate tuning in quantum-dot mode-locked lasers," Appl. Phys. Lett. 104, 021112, (2014).

[10] Habruseva, T., O'Donoghue, S., Rebrova, N., Reid, D. A., Barry, L., Rachinskii, D., Huyet, G., and Hegarty, S. P., "Quantum-dot mode-locked lasers with dual-mode optical injection," IEEE Photon. Technol. Lett. 22 (6), 359-361 (2010).

[11] Habruseva, T., Huyet, G., and Hegarty, S. P., "Dynamics of quantum-dot mode-locked lasers with optical injection,'IEEE J. Sel. Top. Quant. Elec., 17, 1272 (2011).

[12] Habruseva, T., Rebrova, N., Hegarty, S. P., and Huyet, G., [Quantum dot devices, Lecture notes in nanoscale science and technology 13], Springer-Verlag, New York, 65-91 (2012). 